\title{
Shi Xiao San ameliorates the development of adenomyosis in an ICR mouse model
}

\author{
JUAN YE $^{1,2}$, XUETING CAI $^{1,2}$, DAWEI WANG ${ }^{1,2}$, FURONG ZHANG $^{1}$, ZHIGANG WANG $^{1,2}$, MENG CAO $^{1,2}$,

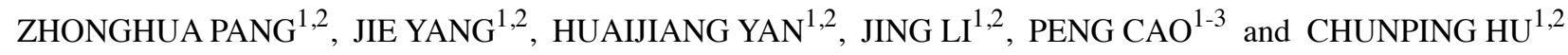 \\ ${ }^{1}$ Affiliated Hospital of Integrated Traditional Chinese and Western Medicine, Nanjing University of Chinese Medicine; \\ ${ }^{2}$ Laboratory of Cellular and Molecular Biology, Jiangsu Province Academy of Traditional Chinese Medicine, \\ Nanjing, Jiangsu 210028; ${ }^{3}$ College of Pharmacy, Nanjing University of Chinese Medicine, \\ Nanjing, Jiangsu 210023, P.R. China
}

Received March 29, 2019; Accepted March 24, 2020

DOI: $10.3892 /$ etm.2020.8994

\begin{abstract}
The use of Shi Xiao San (SXS), composed of Pollen Typhae Angustifoliae and Faeces Trogopterori, can be traced back to the Song dynasty. Traditionally, SXS has been used to treat irregular menstruation, pelvic pain, progressive dysmenorrhea, and postpartum lochiorrhea. The management of adenomyosis (AM) is challenging and to the best of our knowledge there are currently no effective therapeutic strategies. Therefore, the aim of the present study was to investigate the effect of SXS on the development of adenomyosis in a mouse model. AM was induced in 60 neonatal female ICR mice by administering tamoxifen; 10 randomly selected mice were used for model identification via histopathological examination and 10 mice treated with the solvent alone were used as the normal controls. A total of sixty days after birth, the mice treated with AM were randomly divided into four groups and administered one of the following treatments: Low-dose SXS $(55 \mathrm{mg} / \mathrm{kg})$; high-dose SXS (110 mg/kg); danazol ( $1 \mathrm{mg} / 20 \mathrm{~g}$ body weight); or no treatment (model group); at the same time, the normal control group received no treatment. After 2 months of treatment, hotplate and tail-flick tests were used to assess the response to noxious thermal stimuli in the mice, and plasma samples were collected to measure corticosterone levels. Hematoxylin and eosin staining scores of myometrial infiltration and the number of AM nodules were evaluated. Furthermore, the expression of genes associated with AM-related pain was also analyzed. The results from the present study indicated that treatment with SXS decreased myometrial infiltration, alleviated generalized hyperalgesia, and lowered plasma corticosterone levels in mice with induced
\end{abstract}

Correspondence to: Professor Chunping $\mathrm{Hu}$, Laboratory of Cellular and Molecular Biology, Jiangsu Province Academy of Traditional Chinese Medicine, 100 Shizi Street, Hongshang Road, Nanjing, Jiangsu 210028, P.R. China

E-mail: 634068638@qq.com

Key words: Shi Xiao San, adenomyosis, infiltration, hyperalgesia
AM. These findings suggest that SXS effectively attenuated the development of AM, and may serve as a promising treatment approach for AM treatment.

\section{Introduction}

Adenomyosis (AM) is a common gynecological disorder defined by the presence of heterotopic endometrial glands and stroma in the myometrium with adjacent smooth muscle hyperplasia $(1,2)$. Patients with AM often complain of menorrhagia, dysmenorrhea, and subfertility (3). Despite the widespread prevalence of AM, the precise etiology and pathogenesis of AM remains poorly understood (4). Although AM is recognized as a hormone-sensitive disorder, the use of progestogenic agents for treatment has failed to show satisfactory efficacy, indicating the possibility of progesterone resistance (5). Other therapeutic options include gonadotrophin-releasing hormone agonists (GnRH-a), as anti-oestrogenic therapeutic agent, can inhibit AM progression (6); however, the use of GnRH-a is limited to a short-term treatment due to its side effects and recurring symptoms after discontinuation of GnRH-a therapy $(7,8)$. The management of chronic AM is currently a major challenge, with hysterectomy or adenomyomectomy being the frequent treatment of choice (9). Surgery is not a preferred treatment option, especially for patients who wish to remain fertile (10). However, despite the clinical importance of AM, there is little evidence on which to base treatment decisions (11). Therefore, numerous patients with AM turn to alternative treatment modalities, such as Traditional Chinese Medicine (TCM).

Shi Xiao San (SXS), a classical Chinese herbal formula, originated in the Song Dynasty. Based on the fundamental theories of Chinese medicine and clinical experience, SXS is prescribed by herbalists to relieve clinical symptoms resulting from blood stasis, including irregular menstruation, pelvic pain, progressive dysmenorrhea, and postpartum lochiorrhea. Furthermore, patients are able to tolerate long-term SXS therapy (12-14). In Chinese medicine, 'blood stasis' or 'blood stagnation' is considered the main pathogenesis of $\mathrm{AM}$, causing the formation of adenomyotic lesions leading to endometriosis $(15,16)$; therefore, it is suggested that enhancing blood circulation and removing stasis is the basic treatment 
approach for AM $(17,18)$. However, the therapeutic effect of SXS on AM remains unclear.

To date several animal models of AM have been developed. The exposure of neonatal ICR mice to tamoxifen yields the classical model of AM (19-21). Feeding neonatal mice with tamoxifen is a much simpler method compared with using ectopic grafting of pituitary glands, as it requires no surgical skills and has a high induction success rate (22). Furthermore, the classic AM mouse model has an aggravated pathogenic condition with age, including increased myometrial infiltration depth and progressive generalized hyperalgesia (23-25). Finally, this model is not associated with trauma and mice have relatively normal sex hormone levels in their blood; however, this may cause endometrial hormone imbalance (26). Although this is not caused by trauma, it is important for adenomyosis pain research, as it may affect the accuracy of efficacy studies.

The clinical profiles of several premenopausal women with AM, who underwent a hysterectomy, have shown that dysmenorrhea is the most common side effect, often accompanied by menorrhagia (27). It is reported that a higher myometrial infiltration depth is associated with more severe dysmenorrhea in AM (28). Other studies have reported that AM-associated symptoms are variable, non-specific, and related to other associated pathological conditions such as dysfunctional uterine bleeding, leiomyomas and endometriosis $(11,29)$. The factors that determine the frequency and severity of dysmenorrhea in AM remain unknown. No reliable biomarkers for dysmenorrhea or its severity in AM have been identified. A previous study reported that immunoreactivity to both the oxytocin receptor (OXTR) and the transient receptor potential vanilloid type 1 (TRPV1) were positively correlated with the severity of dysmenorrhea and were significant independent predicators for the severity of dysmenorrhea (30). Subsequent research indicated that overexpression of OXTR protein in myometrial smooth muscle cells, that were derived from uterine tissues of patients with AM may be responsible for increased uterine contractibility and AM-associated dysmenorrhea (31). Hence, the specific inhibition of OXTR and Trpv1 may yield a promising therapeutic treatment in the near future.

A previous case-control study found that women with AM were more likely to have a history of depression, or were at higher risk for depression, and therefore were also taking antidepressants (32). Chronic stress is a primary cause of depression and it is well-known that pain is a physical stress factor (33). Therefore, AM-induced chronic pain may result in an elevated physiological arousal that is often associated with the release of stress hormones, such as cortisol in humans or corticosterone (CORT) in rodents. Furthermore, AM has been shown to cause elevated systemic CORT levels in conjunction with generalized hyperalgesia, which was suggestive of increased stress (19).

In the present study, a model of AM was established in ICR mice and the effect of SXS was evaluated on the development of AM to validate our hypothesis that SXS could delay the myometrial infiltration of endometrial implants, alleviate generalized hyperalgesia, and lower stress levels in mice with AM. The current study will be conducive to the development and promotion of SXS as a clinical treatment for AM.

\section{Materials and methods}

Chemicals. Tamoxifen citrate was purchased from Fudan Forward Pharmaceutical Co., Ltd. Danazol capsules were purchased from Jiangsu Lianhuan Pharmaceutical Co., Ltd. Danazol (200 mg) was suspended in phosphate-buffered saline (pH 7.2) at a concentration of $10 \mathrm{mg} / \mathrm{ml}$ and was homogenized using ultrasound in an ice bucket at $400 \mathrm{w}$ for $30 \mathrm{~min}$ (9 sec on, $5 \mathrm{sec}$ off) before intragastric administration.

SXS preparation. The composition of SXS is listed in Table I. All medicinal plants used to prepare the formula were provided and identified by the Department of Pharmacy at the Affiliated Hospital of Integrated Traditional Chinese and Western Medicine, Nanjing University of Chinese Medicine. Individual herbs were deposited in the herbarium for preservation at the Laboratory of Cellular and Molecular Biology, Jiangsu Province Academy of Traditional Chinese Medicine (Nanjing, JiangSu, China). The method used for the preparation of the SXS decoction was as follows: The Chinese medicinal herb, Faeces Trogopterori, was first ground into a fine powder using rice wine (Sha Zhou You Huang; Zhangjiagang Wine Co., Ltd.) and Pollen Typhae Angustifoliae was processed via stir-frying. This was followed by preparation of high-dose SXS, where equal parts of Faeces Trogopterori and Pollen Typhae Angustifoliae were mixed and soaked in $182 \mathrm{ml}$ rice wine for 3 days at room temperature. Meanwhile, low-dose SXS was prepared by diluting the high-dose SXS with an equal volume of rice wine (high-dose SXS volume: rice wine, 1:1). The SXS and its preparations were standardized, regulated, and quality controlled (QC) according to the guidelines defined by the Chinese State Food and Drug Administration (standard no. WS3-B-1912-95). The QC consists of a regular quality check of the herbal medicines, which is performed according to the product specifications for identity, purity, and content of characterizing compounds, thereby ensuring the safety, efficacy, and quality of herbal medicines and their preparations (34).

SXS analysis using high performance liquid chromatography (HPLC). Faeces Trogopterori (1.5 g) and Pollen Typhae (1.5 g) were extracted twice $(2 \mathrm{~h} /$ time $)$ under reflux with $150 \mathrm{ml}$ of yellow rice wine. The extracted solutions were combined, concentrated, and dried at $50^{\circ} \mathrm{C}$ in a vacuum. The dried extract was resuspended in $25 \mathrm{ml}$ methanol then subsequently filtered through a $0.22 \mu \mathrm{m}$ membrane and $20 \mu \mathrm{l}$ of the filtrate was analyzed using HPLC. The internal standards were protocatechuic acid, typhaneoside- reference material, amentoflavone and isorhamnetin.

The HPLC analysis was performed on a serial system (Agilent 1100; Agilent Technologies, Inc.). Briefly, $10 \mu \mathrm{l}$ samples were separated on a reverse-phase analytical column (Zorbax XDB-C18; 4.6x150 mm, 5 mm; Agilent Technologies, Inc.). The mobile phase consisted of solvents $A$ (acetonitrile) and $\mathrm{B}(0.1 \%$ aqueous acetic acid, v/v) with a linear gradient elution as follows: $\mathrm{B}, 0-25 \mathrm{~min}$ at $3-10 \%$; $\mathrm{B}, 25-40 \mathrm{~min}$ at $10-15 \%$; A, $40-65 \mathrm{~min}$ at $30 \%$; B, $65-70 \mathrm{~min}$ at $100 \%$. The flow rate was $1 \mathrm{ml} / \mathrm{min}$ with a column temperature of $30^{\circ} \mathrm{C}$. The experiment was performed on a serial System (LC-20AT HPLC system; Shimadzu Corporation) with an SPD-20A 
Table I. The composition of Shi Xiao San.

\begin{tabular}{lccccc}
\hline Ingredients (Latin name) & Chinese name & Medicinal parts & Origin & Grams & $\%$ \\
\hline Pollen Typhae Angustifoliae & Puhuang & Pollen & Jiangsu, China & 9 & 50 \\
Faeces Trogopterori & Wulingzhi & Feces & Hebei, China & 9 & 50 \\
Total amount & N/A & N/A & N/A & 18 & 100 \\
\hline
\end{tabular}

N/A, not applicable.

detector (Shimadzu Corporation) using validated methods for linearity and quantification. A Shimadzu diode array detector (Shimadzu Corporation) was set at $280 \mathrm{~nm}$ to detect the constituents of SXS.

Animals and treatments. A total of 15 separate litters of newborn (day 1 after birth; born at the same time point) ICR mice and their mother were purchased from Shanghai Laboratory Animal Corporation. The mother mouse together with their respective offspring were housed in the same cage under the following controlled conditions: $\sim 25^{\circ} \mathrm{C}, 12: 12$ light/dark cycle with lights on at 6:00 in the morning and had ad libitum access to chow and fresh water (20). A total of $1 \mathrm{mg} / \mathrm{kg}$ tamoxifen suspended in a peanut oil/lecithin/condensed milk mixture [2:(0.2):3, by volume] was orally administered to female neonatal mice at $5 \mu \mathrm{l} / \mathrm{g}$ body weight (bw) from days 2 to 5 after birth. A total of 10 randomly designated control female neonatal mice were fed similarly, however they were not administered with tamoxifen. At 3 weeks, offspring were weaned and separated from their mothers. A total of 60 days after birth, 10 treated and 10 untreated mice were used to detect the incidence of AM using hematoxylin and eosin (H\&E) staining. All experiments were approved by the Institutional Experimental Animals review board of the Affiliated Hospital of Integrated Traditional Chinese and Western Medicine, Affiliated to Nanjing University of Chinese Medicine Ethics Review Board.

Experimental protocol. Female neonatal mice were given $1 \mathrm{mg} / \mathrm{kg}$ tamoxifen orally, from days 2 to 5 after birth. A total of 60 days after birth, 10 randomly selected mice were used for model identification via histopathological examination, while 10 neonatal mice, which were treated similarly with the solvent alone, served as controls. The remaining mice treated with tamoxifen were randomly divided into five, equal-sized groups, with each receiving a different treatment via daily intragastric administration for 2 months, after the AM model was successfully established. Successful establishment was confirmed via HE examination of uterine tissue sections, which exhibited endometrial infiltration into the muscle layer in mice. The five groups were as follows: The model $(\mathrm{M})$ group $(\mathrm{n}=10)$ received the vehicle only (sterile water); the positive $(P)$ group $(n=10)$ received danazol $(1 \mathrm{mg} / 20 \mathrm{~g}$ bw) treatment; the low-dose (L) group $(\mathrm{n}=10)$ received a low-dose $(55 \mathrm{mg} / \mathrm{kg}$ bw/day) SXS treatment; the high-dose $(\mathrm{H})$ group $(\mathrm{n}=10)$ received a high-dose (110 mg/kg bw/day) SXS treatment; and the control (C) group $(n=10)$ consisted of neonatal mice treated vehicle alone (PBS) and served as controls. The doses of the drugs used in the present study were determined based on the doses used in humans converted to that used in mice, using the formula given in previous reports $(22,35,36)$. Following 2 months of SXS treatment, the hotplate and tail-flick test were performed in all groups. Mice were randomly selected for hyperalgesia measurements. Hotplate test and tail-flick tests were separately performed with a $>30$ min interval. The final body weight was measured and peripheral blood was collected subsequent to all mice being euthanized using cervical dislocation. Mice were weighed prior to sacrifice, and uterine weight was subsequently recorded during dissection to calculate the ratio of the uterine wet weight to total body weight (U/B). Blood collection from orbital venous plexus was performed before sacrifice, both uterine horns were harvested, and the uterine weight was recorded. The contour of the uterus was examined immediately with the naked eye and adenomyotic nodules extruding from the surface of the uterus were counted under a magnifier (magnification, x50 and x200). The left uterine horn was snap-frozen in liquid nitrogen and stored at $-80^{\circ} \mathrm{C}$ until further analysis, and the right uterine horn was fixed in $4 \%$ paraformaldehyde and embedded in paraffin at room temperature for $<1$ week for subsequent histopathological studies. Subsequently, H\&E staining was performed to evaluate the depth of myometrial infiltration of the ectopic endometrium, according to the standard of Mori's classification, which grades the progression of adenomyosis. The grading system was as follows: Grade 0, normal uterus; grade 1, uterus with endometrial stromal cell invasion into the inner layer of the myometrium; grade 2, uterus with endometrial stromal and gland cell invasion into the inner layer of the myometrium; grade 3 , uterus with endometrial stromal and gland cell invasion into the connective tissue space between the inner and outer myometrial layers; grade 4, uterus with cystic hyperplasia of the endometrial glands and small nodules beneath the serosa; grade 5, uterus with cystic hyperplasia of the endometrial glands and a large number of subserosal nodules (37).

Histopathological examination. H\&E staining was performed as described in our previous report (38). Briefly, serial $4-\mu \mathrm{m}$ sections were obtained from each paraffin-embedded tissue block, and subsequently three randomly selected sections were deparaffinized for $15 \mathrm{~min}$, and rehydrated in descending alcohol series $(100,95$, and $70 \%)$ for $5 \mathrm{~min}$ each at room temperature. The sections were washed briefly with distilled water, stained in hematoxylin solution for $8 \mathrm{~min}$ at room temperature, and washed again with running tap water for $5 \mathrm{~min}$. The sections were then differentiated in $1 \%$ acid alcohol for $30 \mathrm{sec}$ and counterstained with eosin solution for $30 \mathrm{sec}$. Following dehydration using $95 \%$ alcohol for $5 \mathrm{~min}$, the sections were air-dried and 
mounted. All images were obtained with an Olympus BX51 microscope (magnification, x50 and x200) fitted with a digital camera (Olympus Corporation). For histological examination, three randomly selected sections of each mouse were chosen for $H \& E$ staining to confirm the pathological diagnosis. If the endometrial stroma with or without gland was observed to be displaced in the myometrium, the diagnosis of AM was considered to be confirmed. Sections were scored by two blinded pathologists at the Department of Pathology, Affiliated Hospital of Integrated Traditional Chinese and Western Medicine, Nanjing University of Chinese Medicine (Jiangsu, China). The H\&E scoring was performed in accordance with previously published studies (39-41).

Hotplate and tail-flick tests. The hotplate test is a classic method for measuring the latent period preceding pain and nociception in rodents, for the evaluation of analgesic drugs. It is also a test of the response threshold to thermal stimuli, representing a form of acute thermal pain. In the present study, the hotplate test was performed with the Hot Plate Analgesia Meter (BME-480; Jiangsu Province Academy of Traditional Chinese Medicine, China) consisting of a metal plate $(25 \times 25 \mathrm{~cm})$ with a plastic cylinder ( $20 \mathrm{~cm}$ in diameter; $18 \mathrm{~cm}$ in height). The surface of the plate can be heated to a constant temperature of $50.0( \pm 0.1)^{\circ} \mathrm{C}$ as measured using a built-in digital thermometer and a plastic cylinder. Mice were brought to the testing room and allowed to acclimatize for $5 \mathrm{~min}$ prior to the test. The response latency to a noxious thermal stimulus is defined as the time(s) elapsed from the moment when the mouse was inserted inside the cylinder to the point when it licked its hind paws.

The tail-flick test was evaluated with a commercially available tail-flick Analgesia Meter (33T; NatureGene Corporation). Thermal stimulation was provided using a beam of high-intensity light focused $1-2 \mathrm{~cm}$ distal to the tail end. The time between the start of the stimulation and tail withdrawal was measured as the tail-flick latency. The intensity of the beam was adjusted to produce a mean control reaction time within $60 \mathrm{sec}$ prior to the initiation of the test, after which the heat source was held constant $\left(59^{\circ} \mathrm{C}\right)$. Mice were then placed in a metallic cage with their tails extended through a slot located at the rear of the cage and allowed to acclimatize for $10 \mathrm{~min}$ prior to the test. Withdrawal of the tail exposed to the light instantly turned off the thermal stimulus, automatically stopped the internal timer, and the latency time was automatically displayed. For the hotplate and tail-flick tests, mice were tested only once per session, separately. The latency was calculated as the mean of three recorded readings at $12 \mathrm{~h}$ intervals.

$R N A$ extraction and reverse transcription-quantitative PCR (RT-qPCR). The mRNA levels of OXTR and Trpv1 were determined using RT-qPCR analysis. Total RNA from uterine tissues was isolated with TRIzol ${ }^{\circledR}$ (Invitrogen; Thermo Fisher Scientific, Inc.). Complementary DNA (cDNA) was synthesized from $1 \mu \mathrm{g}$ RNA using the Maxima first-strand cDNA synthesis kit (Fermentas; Thermo Fisher Scientific, Inc.), according to the manufacturer's protocols. RT-qPCR was performed using a 7500 Real-Time PCR System (Applied Biosystems; Thermo Fisher Scientific, Inc.) and 2X Maxima SYBR Green/ROX qPCR master mix (Fermentas; Thermo Fisher Scientific, Inc.) according to the manufacturer's protocols. The primers used were as follows: OXTR forward, 5'-CAGACGCAGAGTGGTGCTAA-3' and reverse, 5'-CCTTTCAGCCCTCGTTCTCA-3'; Trpv1 forward, 5'-CCACTCTTCTCCCACACGAG-3' and reverse, 5'-GGCAGGTGTCCTTTTGGAGT-3'; GAPDH forward, 5'-CTCССАСТСТТССАССТTCG-3' and reverse, 5'-CCACCACCCTGTTGCTGTAG-3'. The thermocycling conditions were as follows: Initial denaturation at $95^{\circ} \mathrm{C}$ for $5 \mathrm{~min}$; followed by $15 \mathrm{sec}$ at $95^{\circ} \mathrm{C}$ and $30 \mathrm{sec}$ at $60^{\circ} \mathrm{C}$ for 35 cycles. The relative transcription level was calculated using $2^{-\triangle \Delta C q}(35,42,43)$ taking GAPDH as the internal standard control. Five samples from each group were analyzed in triplicate.

Measurement of plasma CORT level using ELISA. The CORT ELISA kit (cat. no. ml037564) was purchased from Shanghai Enzyme-linked Biotechnology Co., Ltd. with a sensitivity limit of $2 \mu \mathrm{g} / \mathrm{ml}$ for CORT and measurements were performed following the manufacturer's protocols. Briefly, the harvested blood samples were collected in sterile tubes containing liquid EDTA, which were kept on ice. The samples were centrifuged at 2,500 x g for $10 \mathrm{~min}$ at $4^{\circ} \mathrm{C}$ and with use of an anticoagulation tube with $1 \mathrm{mg} / \mathrm{ml}$ EDTA, the plasma was harvested from the orbital venous plexus and stored at $-80^{\circ} \mathrm{C}$ until use. When ELISA assay was performed, the absorbance was read immediately using a microplate reader (Thermo Fisher Scientific, Inc.) at a wavelength of $450 \mathrm{~nm}$ and the mean optical density was converted into concentration. Five samples from each group were evaluated in triplicate. The coefficients of variation were $<5 \%$ and $\mathrm{r}^{2}$ of the standard curve for the assay was $>0.98$.

Statistical analysis. SPSS 19.0 software (IBM Corp.) and GraphPad Prism 5.01 (GraphPad Software Inc.) were used for statistical analysis. The data are presented as the mean \pm standard error of mean or medians unless otherwise indicated. Differences among multiple groups were evaluated via one-way analysis of variance with Tukey's post hoc test to identify the significantly different groups. $\mathrm{P}<0.05$ was considered to indicate a statistically significant difference.

\section{Results}

Successful induction of AM in ICR mice. Hematoxylin and eosin staining revealed that AM was successfully induced in all mice treated with tamoxifen and was absent in untreated mice, consistent with previous reports $(20,21)$. As indicated in Fig. 1C and D, ectopic endometrial tissues invading the myometrium and reaching the uterine serosa appeared in the adenomyotic uteri of mice treated with tamoxifen. In mice without AM, a distinct border between the basal layer of the endometrium and the adjacent myometrium was observed (Fig. 1A and B). At 60 days after birth, the H\&E staining scores in tamoxifen-treated mice and controls were $1.6 \pm 0.3$ and $0.0 \pm 0.0$, respectively.

Effect of SXS treatment on myometrial infiltration in AM-induced mice. HPLC fingerprint spectra for SXS and the main standard components are presented in Fig. 2. The content of standard material in SXS (Fig. 2A), including 3,4-Dihydroxybenzoic acid (Fig. 2B), typhaneoside (Fig. 2C), amentoflavone (Fig. 2D), isorhamnetin (Fig. 2E) complied with 
A

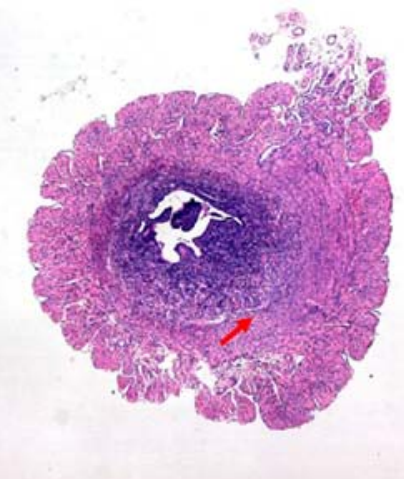

C

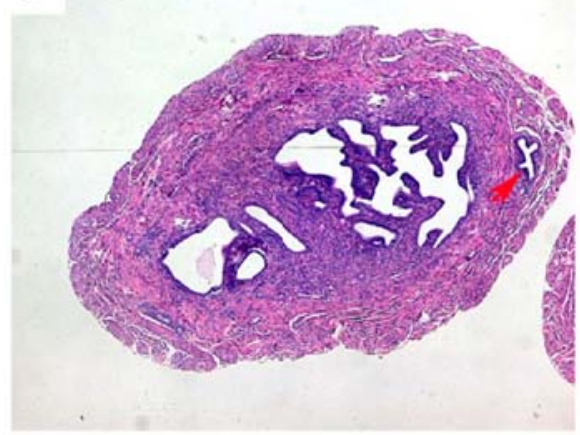

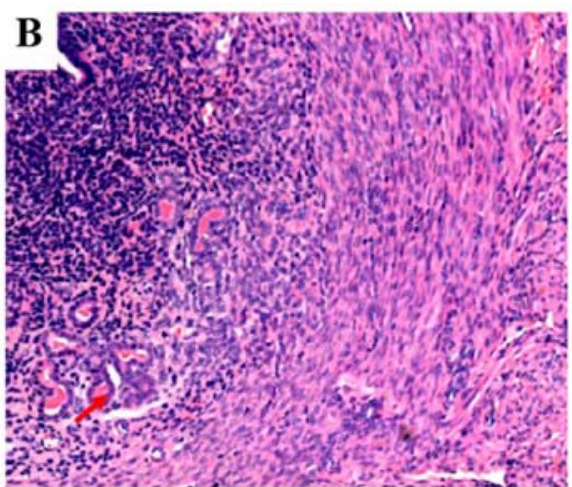

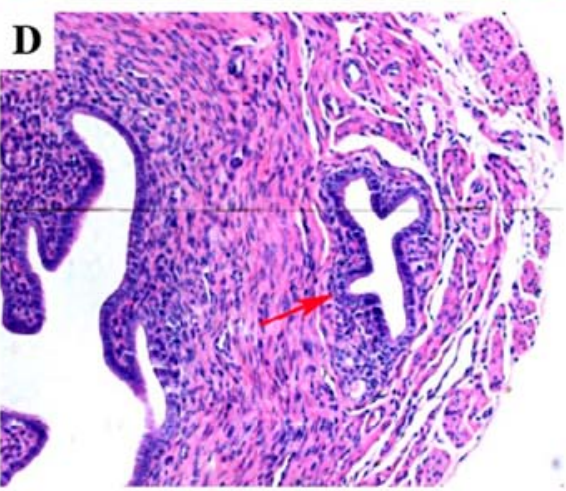

Figure 1. Representative microscopic images of the uteri from mice treated with tamoxifen and control mice without treatment. (A) Uteri (magnification, $\mathrm{x} 50$ ) and (B) uteri (magnification, x200) from mice treated with tamoxifen showing the endometrial lumen lined by columnar cells with well-defined layers of the endometrial stroma and smooth muscle (red arrows, normal myometrium). (C) Uteri (magnification, x50) and (D) uteri (magnification, x200), from untreated mice showing adenomyosis with endometrial glands penetrating deep into the myometrium (indicated with the red arrows).

A

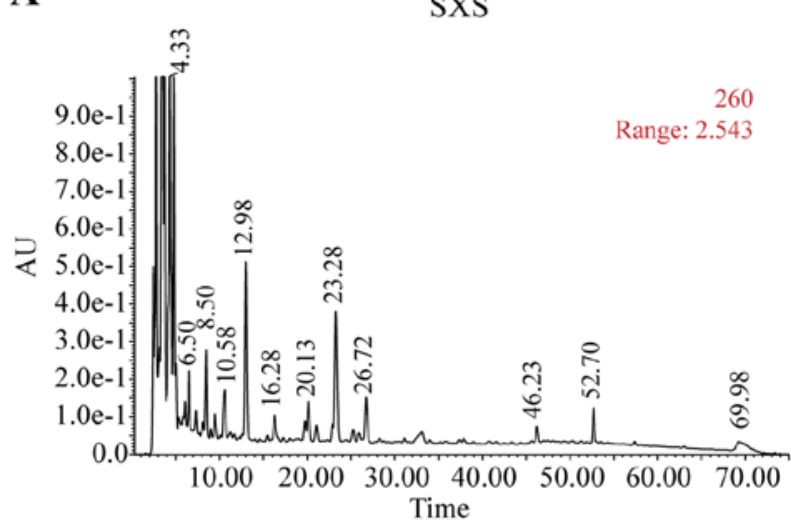

C

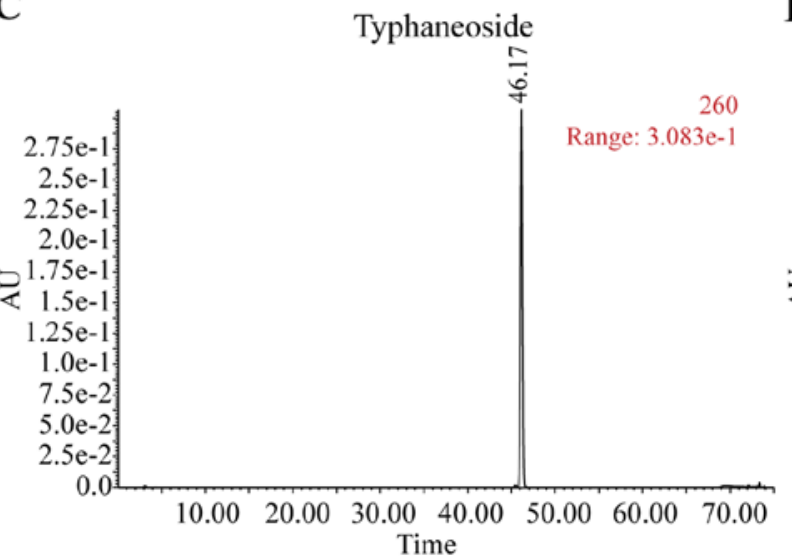

B

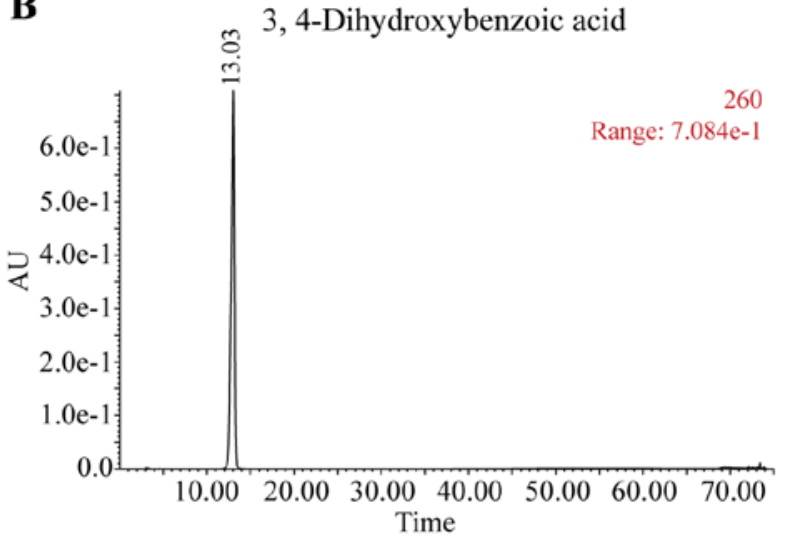

D

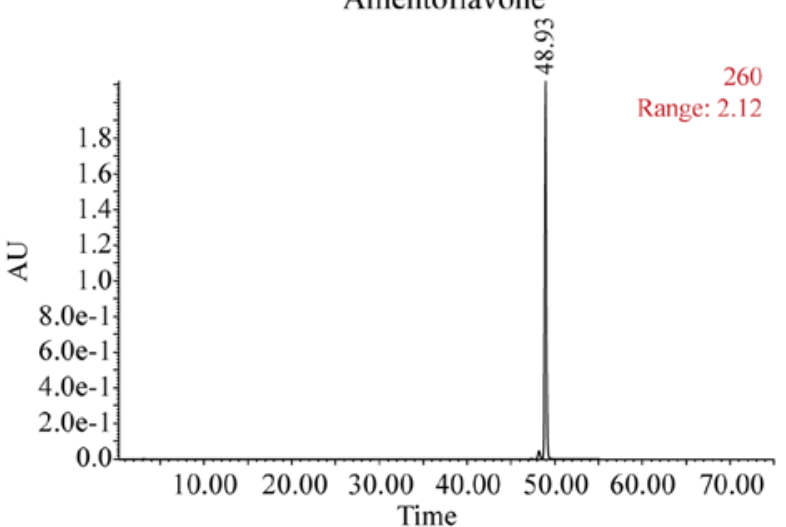

Figure 2. High performance liquid chromatograms of SXS and the primary standard components. (A) SXS; (B) 3,4-Dihydroxybenzoic acid; (C) Typhaneoside; (D) Amentoflavone. 


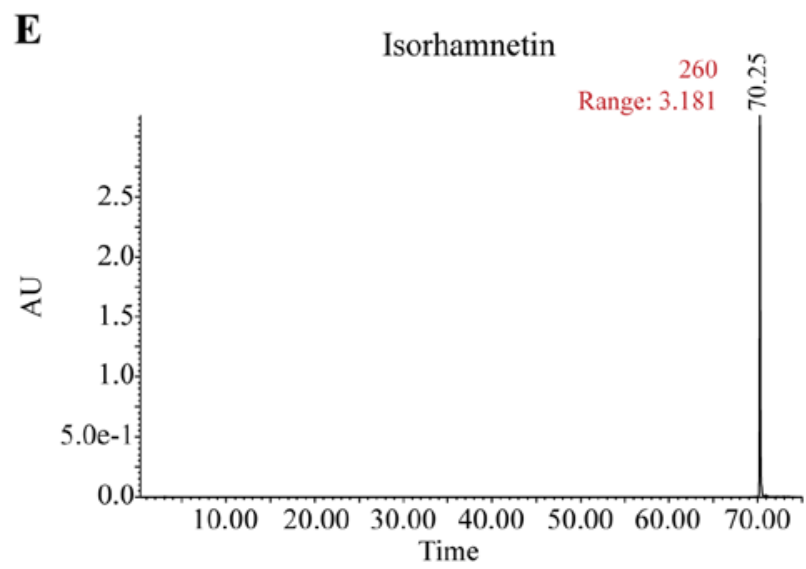

Figure 2. Continued. (E) Isorhamnetin. AU, area under the curve; SXS, Shi Xiao San

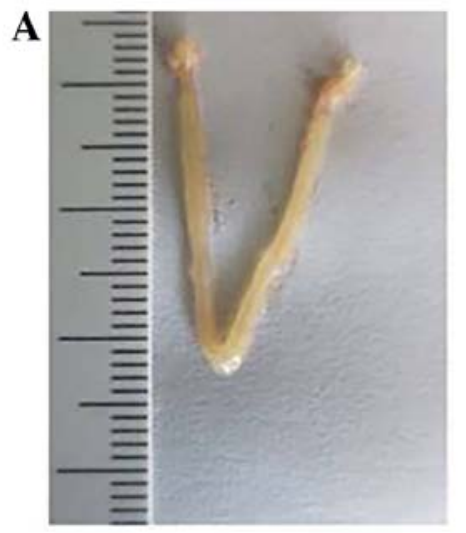

D

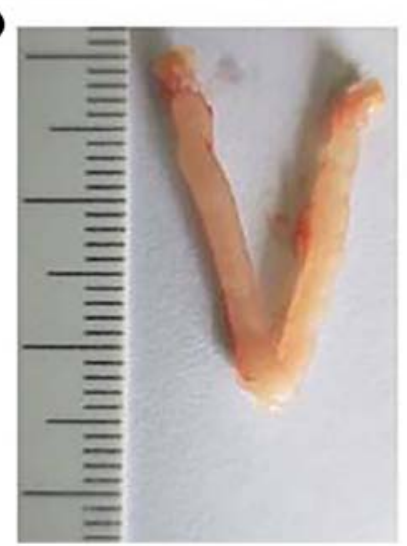

B

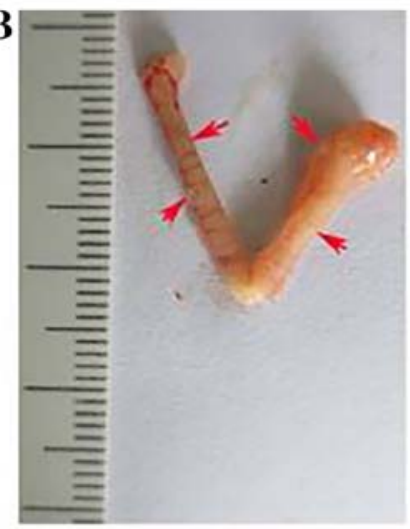

C

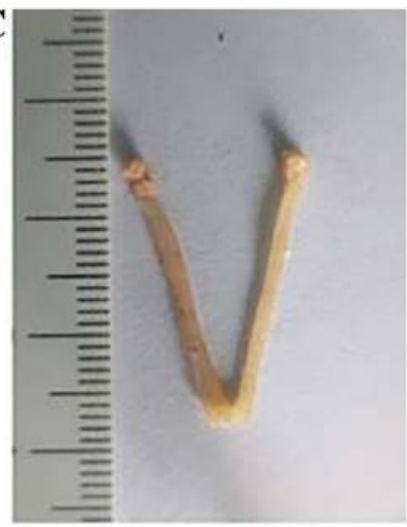

$\mathbf{E}$

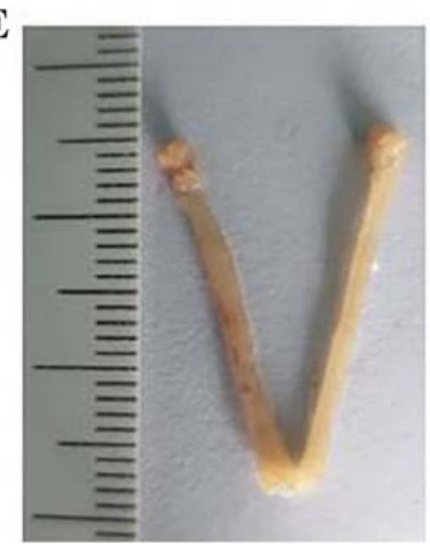

Figure 3. SXS efficacy on AM in an experimentally induced ICR mouse model. (A) Control animals with normal uterine contours, smooth walls, and no congestion. (B) Untreated adenomyotic animals, showing distorted uterine contours and AM nodules extruding from the surface of the uteri. (C) Adenomyotic animals treated with $1.0 \mathrm{mg}$ danazol/20 $\mathrm{g}$ body weight administered intragastrically, showing marked improvement of the distorted uterine contour and extruding AM nodules. (D) Adenomyotic animals treated intragastrically with $100 \mathrm{mg} \mathrm{SXS/kg}$ body weight, showing slight improvement of the distorted uterine contours and extruding AM nodules. (E) Adenomyotic animals treated intragastrically with $55 \mathrm{mg}$ SXS/kg body weight, showing marked improvement of the distorted uterine contour and extruding AM nodules. Red arrows indicate small AM nodules extruding from the serosal surface. AM, adenomyosis; SXS, Shi Xiao San.

Chinese Pharmacopoeia standards. The uteri of the mice in the control group showed normal contours and normal blood flow without noticeable redness (Fig. 3A). The uteri of mice in group $\mathrm{M}$ showed distorted contours and adenomyotic nodules that clearly extruded from the serosal surface (Fig. 3B). The uteri of mice in group $\mathrm{P}$ showed improved uterine contours with no obvious adenomyotic nodules extruding from the serosal surface (Fig. 3C). The uteri from mice in groups $\mathrm{H}$ and L showed improved contours and less congestion compared with that in group $\mathrm{M}$, with changes occurring in a dose-dependent manner. The uterus of the low-dose group is presented in Fig. 3D and the uterus of the high-dose group is demonstrated in Fig. 3E.

As indicated in Table II, the uterine wet weight and the final body weight of the mice in the control and treatment groups were not significantly different. However, the ratio of the uterine wet weight to the total body weight (U/B) was significantly lower in the model group and group L compared 
with that in the control group (both $\mathrm{P}<0.001$ ). In addition no significant differences were observed between the control group and group $\mathrm{P}(\mathrm{P}=0.664)$ or $\mathrm{H}(\mathrm{P}=0.152)$. In addition, the $\mathrm{U} / \mathrm{B}$ ratio of group $\mathrm{H}$ was comparable to that of group $\mathrm{P}$ $(\mathrm{P}=0.313)$.

The number of AM nodules and the H\&E scores in groups $\mathrm{M}$ and $\mathrm{P}$ were $11.2 \pm 0.9,3.3 \pm 0.4$; and $4.4 \pm 0.5,1.5 \pm 0.2$, respectively (Table II). SXS treatment reduced the number of AM nodules and the H\&E scores in a dose-dependent manner. The decrease in the number of $\mathrm{AM}$ nodules and $\mathrm{H} \& \mathrm{E}$ scores was significant in Group $\mathrm{L}(\mathrm{P}<0.001$ and $\mathrm{P}=0.002$, for $\mathrm{AM}$ nodules and $\mathrm{H} \& \mathrm{E}$ scores, respectively) and $\mathrm{H}(\mathrm{P}<0.001, \mathrm{P}<0.001$, for AM nodules and H\&E scores, respectively) compared with that in the model group. In addition, the number of AM nodules in groups $\mathrm{H}$ and $\mathrm{L}$ was compared to the number of AM nodules in group $\mathrm{P}(\mathrm{P}=0.794$ and $\mathrm{P}=0.041$, for group $\mathrm{H}$ and $\mathrm{L}$, respectively). $\mathrm{H} \& \mathrm{E}$ scores of group $\mathrm{P}$ were not significant when compared with that in group $\mathrm{H}(\mathrm{P}=0.38)$ however, they were significant compared with that in group $\mathrm{L}(\mathrm{P}=0.44)$.

Effect of SXS on the thermal response latency. Using the tail-flick test, the present study assessed the response to noxious thermal stimuli in mice after 2 months of SXS treatment (Fig. 4A). In mice treated with danazol (group P) and the low- and high-dose SXS (groups $\mathrm{L}$ and $\mathrm{H}$, respectively), the mean response latency was significantly lower $(\mathrm{P}<0.001)$ compared with that in group $\mathrm{C}$, suggesting that the treatment did not completely restore the response latency to a normal level.

The present study also assessed the response latency in mice using the hotplate test. Unlike the tail-flick latency test, the treatment completely restored the response latency to a normal level in mice treated with danazol, low-dose SXS, and high-dose SXS ( $\mathrm{P}=0.055, \mathrm{P}=0.196$, and $\mathrm{P}=0.960$, for groups $\mathrm{P}, \mathrm{L}$, and $\mathrm{H}$, respectively) compared with that in the control group.

SXS modulates the expression of genes associated with AM-induced pain. Using RT-qPCR, the present study analyzed the expression of genes associated with AM-induced pain. As indicated in Fig. 5A and B, the expression of OXTR and Trpv1 was significantly higher in the uteri of mice in group $\mathrm{M}$ compared with that in group $\mathrm{C}(\mathrm{P}<0.001)$. As shown in Fig. 5A, both low- and high-dose SXS treatment downregulated the mean mRNA expression level of OXTR in the uteri in a dose-dependent manner compared to that in group $\mathrm{M}$. Furthermore, the OXTR expression level in group $\mathrm{H}$ was similar with the control group ( $\mathrm{P}=0.301)$. In addition, SXS treatment also reduced the mRNA expression level of Trpvl in a dose-dependent manner, and this decrease in Trpv1 expression was significantly lower in groups $\mathrm{L}$ and $\mathrm{H}$ compared with that in group $\mathrm{M}(\mathrm{P}=0.001$ and $\mathrm{P}<0.001$, for groups $\mathrm{L}$ and $\mathrm{H}$, respectively). However, the mRNA expression level of Trpv1 in both groups $\mathrm{L}(\mathrm{P}<0.001)$ and $\mathrm{H}(\mathrm{P}=0.010)$ remained significantly higher compared with that in group $\mathrm{C}$.

Effect of SXS on CORT plasma levels. As indicated in Fig. 6, the present study found significant differences in the plasma CORT levels among the five mice groups $\left(\mathrm{P}<1.0 \times 10^{-4}\right)$. In particular, group $\mathrm{M}$ had a significantly elevated CORT expression level when compared with that in the control group

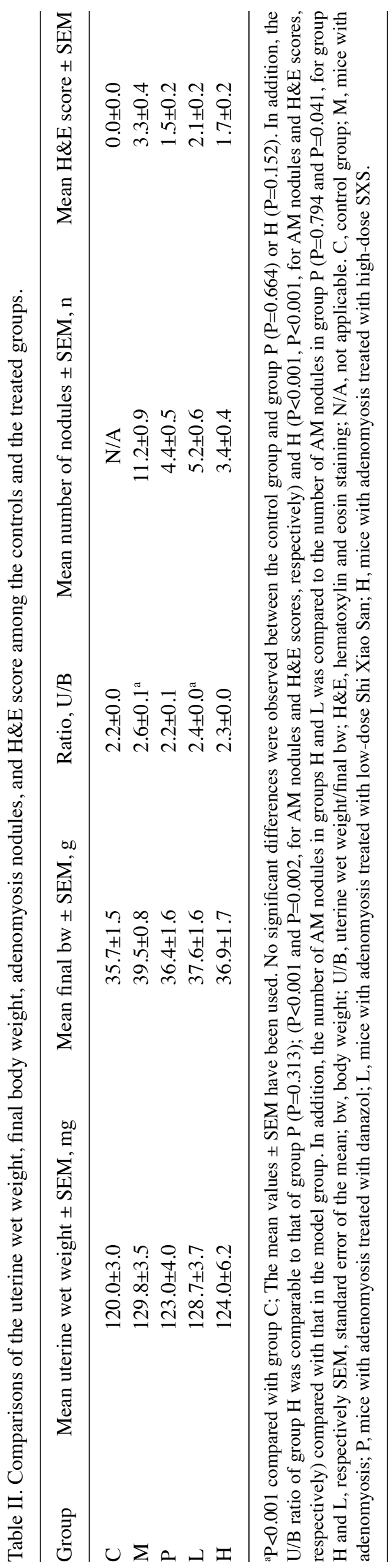



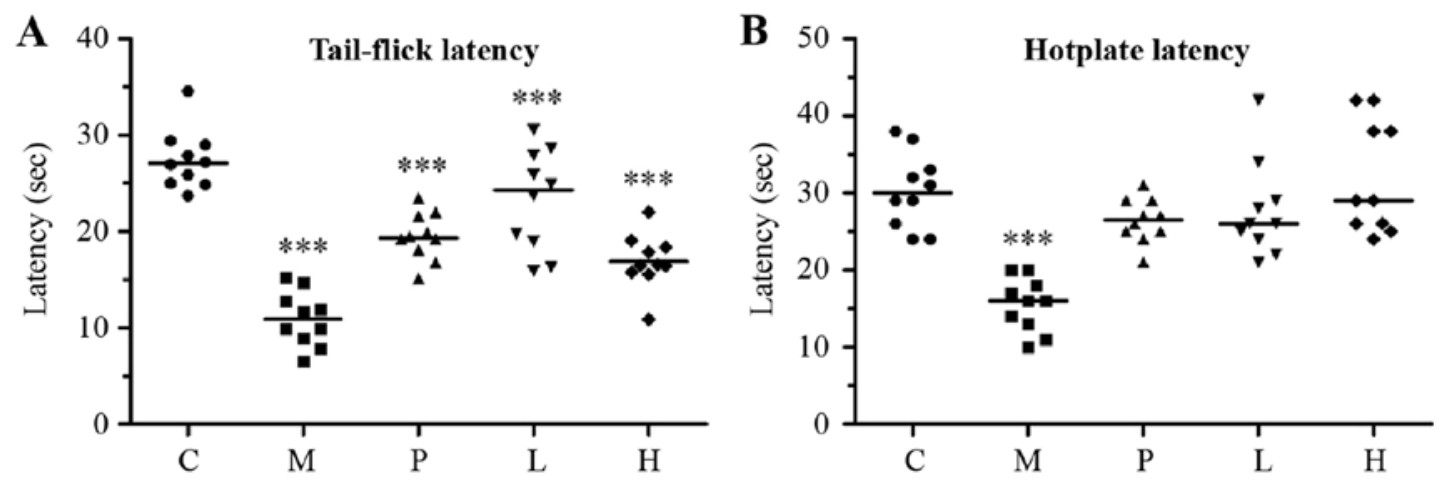

Figure 4. SXS effect on the thermal response latency. Beeswarm plots of (A) tail-flick latency and (B) hotplate in the different treatment groups. ${ }^{* * * *} \mathrm{P}<0.001$ vs. the control group. AM, adenomyosis; SXS, Shi Xiao San; C, control group comprised of mice without AM and received no treatment; M, mice with AM not administered with any other treatment; P, mice with AM and treated with danazol; L, mice with AM treated with low-dose SXS; H, mice with AM and treated with high-dose SXS .

A

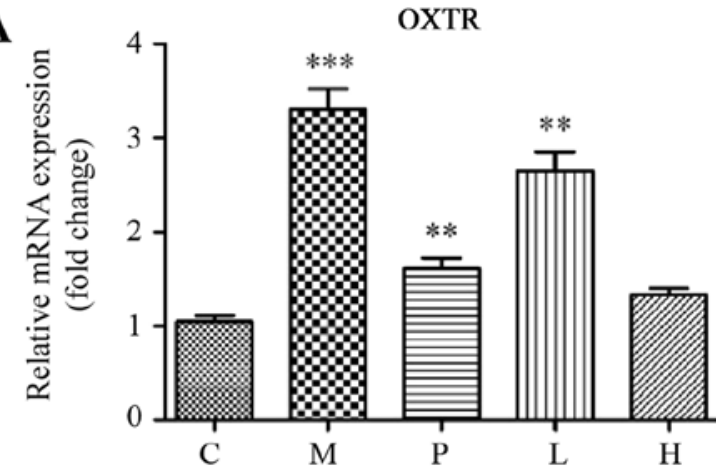

B

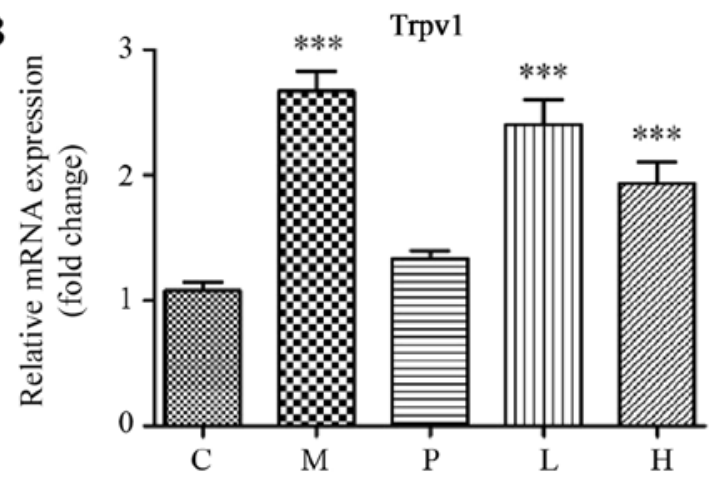

Figure 5. SXS modulates the expression of genes associated with AM-induced pain. The histogram shows relative mRNA expression levels of (A) OXTR and (B) Trpv1 in each treatment group. Bars and whiskers indicate the mean \pm standard error of the mean. Differences between groups were analyzed using Tukey's post hoc test following one-way analysis of variance. ${ }^{* *} \mathrm{P}<0.01$ and ${ }^{* * * *} \mathrm{P}<0.001$ compared with the control group. AM, adenomyosis; SXS, Shi Xiao San; C, control group comprised of mice without AM and received no treatment; M, mice with AM not administered any other treatment; P, mice with AM and treated with danazol; L, mice with AM treated with low-dose SXS; H, mice with AM and treated with high-dose SXS; OXTR, oxytocin receptor; Trpv1, transient receptor potential vanilloid type 1 .

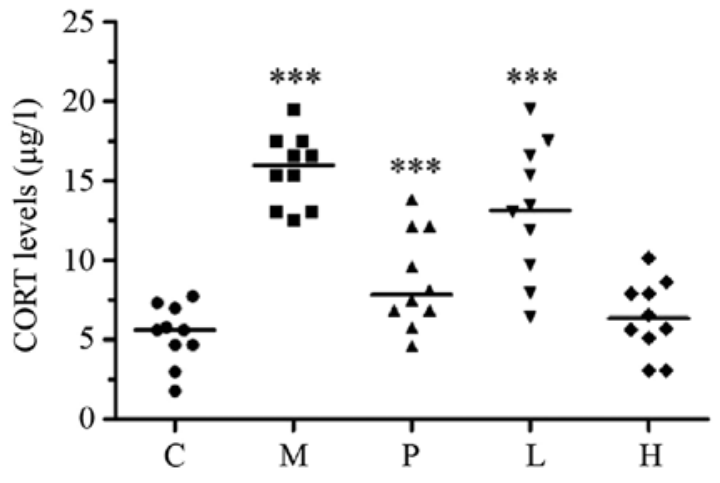

Figure 6. Plasma CORT $(\mu \mathrm{g} / \mathrm{l})$ levels among the different experimental groups. ${ }^{* * *} \mathrm{P}<0.001$ vs. the control group. AM, adenomyosis; SXS, Shi Xiao San; $\mathrm{C}$, control group comprised of mice without AM and received no treatment; M, mice with AM not administered any other treatment; P, mice with $\mathrm{AM}$ and treated with danazol; L, mice with AM treated with low-dose SXS $\mathrm{H}$, mice with AM and treated with high-dose SXS; CORT, corticosterone.

$(\mathrm{P}<0.001)$. The mean CORT level in group $\mathrm{H}$ was similar to that in the control group $(\mathrm{P}=0.613)$, however it was significantly lower compared with that in group $\mathrm{P}(\mathrm{P}=0.010)$. In contrast, the mean CORT level in group $\mathrm{L}$ was similar to that in group $\mathrm{P}$ $(\mathrm{P}=0.912)$ however, it was significantly higher compared with that in the control group $(\mathrm{P}=0.010)$. Furthermore, unlike the model group, both low- and high-dose SXS lowered plasma CORT levels in mice with induced AM, in a dose-dependent manner.

\section{Discussion}

The use of TCM for disease treatment is considered to have low side effects and to be relatively cost-efficient; therefore, TCM is being used more frequently worldwide (44). SXS was designed based on TCM syndrome typing, and followed the rules for drug synergism and compatibility to resolve blood stasis (12-14). In the present study, it was found that SXS could attenuate the development of AM in mice with experimentally-induced AM in a dose-dependent manner.

In previous studies, the area and depth of the ectopic endometrium in the uterus of mice with induced AM continuously increased with age (45-48). Consistent with the aforementioned, the present study conducted a comparison of $\mathrm{H} \& \mathrm{E}$ staining scores of tamoxifen-treated mice at 60 and 120 days following birth. The results revealed a progressive infiltration of the endometrium. SXS treatment showed a dose-dependent suppression of myometrial infiltration. Based on a comparison of AM nodules and H\&E scores for groups $\mathrm{H}$ and $\mathrm{P}$ (danazol 
treatment), it can be concluded that SXS treatment and danazol oral administration had almost equivalent efficacy in this model.

The induction of AM in ICR mice results in progressive generalized hyperalgesia, similar to that in humans, along with elevated amplitude and increased irregularity of uterine contractions (19-21). The present study evaluated the response of mice to thermal stimuli using the hotplate and tail-flick tests after 2 months of SXS treatment, which confirmed the results of previous reports that exposure of neonatal ICR mice to tamoxifen could induce generalized hyperalgesia $(19-21,43,49)$. Furthermore, it was demonstrated that treatment with SXS improved the generalized hyperalgesia, although the response latency in the tail-flick test was not completely restored to the normal level. Nevertheless, the effect of SXS appeared to be dose-dependent in both the hotplate and tail-flick tests. In brief, these results indicated that SXS was a promising compound for the treatment of AM-induced hyperalgesia, especially considering the low incidence of side effects and the cost-efficiency of treatment.

OXTR expression has previously been reported to be increased in the epithelial, however, not in the stromal cells and in the smooth muscle cells in endometriotic lesions (42). TRPV1 is known to be a key molecule in sensory nerves in peripheral nociception integrating multiple pain-producing stimuli (43). A previous study revealed that immunoreactivity of OXTR and TRPV1 was significantly higher in the ectopic endometrium of women with AM compared with women with a healthy endometrium, and that both OXTR and TRPV1 immunoreactivity positively correlated with the severity of dysmenorrhea; therefore, they are considered significant predictors of AM (30). TRPV1 activation may trigger the release of neuropeptides, such as substance $\mathrm{P}$ and calcitonin gene-related peptide, causing increased blood flow and hyperalgesia and perhaps dysmenorrheal (50). Therefore, TRPV1 could be an important therapeutic target for adenomyosis-associated dysmenorrhea in light of the recent development in TRPV1 antagonists (51).

The data from the present study suggests that SXS was a potent inhibitor of OXTR and Trpv1. However, owing to technical limitations, the expression of genes associated with AM-induced pain from the entire uterus could be investigated. It is expected that the response of the endometrial, stromal, and myometrial components will differ. If proteins are only expressed in different cell types, the extent of the changes in these proteins may be larger compared with that identified from analyzing the whole organ. Techniques, such as laser capture microdissection are required to examine cell-specific responses to SXS.

Irrespective of the cause, the AM-induced elevation of plasma CORT levels observed in the present study is consistent with several studies, which have reported that chronic pain conditions, such as rheumatoid arthritis, induce stress in patients and are associated with dysfunction of the hypothalamic-pituitary-adrenal axis. In turn, this may exacerbate the symptoms of chronic pain $(52,53)$. The results from the present study, together with a previous report that pre-induction stress exacerbates AM (54), has raised the possibility that stress and AM may be mutually facilitative, thereby perpetuating AM-induced pain. Furthermore, SXS treatment was found to be effective in reducing systemic CORT levels in the present study.

Aside from abnormal uterine bleeding, dysmenorrhea is the most prevalent symptom of AM, and is arguably the most debilitating symptom (17). The data from the present study suggests that SXS alleviated generalized hyperalgesia in the AM mouse model through the regulation of the expression of pain-associated genes and stress levels. However, it remains to be elucidated whether the ability to regulate these genes can be considered a primary or a secondary therapeutic target for AM.

Collectively, the present study has demonstrated that treatment with SXS reduced myometrial infiltration, alleviated generalized hyperalgesia, and decreased plasma CORT levels in mice with induced AM. In addition, SXS tolerance, demonstrated in the AM mouse model, was consistent with that of long-term SXS therapy for patients with AM in the clinical setting (12-14). From the perspective of Chinese medicine, SXS is a promising formula for the treatment of AM, owing to the favorable associated costs and side-effect profile $(55,56)$. However, caution should be exercised, as a successful therapy for animals does not guarantee an effective therapy for humans. Thus, further research is required to allow a comprehensive examination of the potential benefits of SXS.

\section{Acknowledgements}

Not applicable.

\section{Funding}

This study was supported by the National Natural Science Foundation of China (grant no. 81674013), Jiangsu Province's Outstanding Leader Program of Traditional Chinese Medicine, and the Natural Science Foundation of Jiangsu Province (grant no. BK20161607) and Science and technology project of Jiangsu Provincial Administration of traditional Chinese Medicine (grant no. YB2015049).

\section{Availability of data and materials}

The datasets used and/or analyzed during the present study are available from the corresponding author on reasonable request.

\section{Authors' contributions}

$\mathrm{CH}, \mathrm{PC}$ and JuY designed the research. FZ, DW, ZW, ZP, JiY and $\mathrm{HY}$ performed the experiments. XC, MC and JL analyzed the data. JuY wrote the manuscript. All authors read and approved the final version of this manuscript.

\section{Ethics approval and consent to participate}

This study was approved by the Hospital of Integrated Traditional Chinese and Western Medicine Affiliated to Nanjing University of Chinese Medicine Ethics Review Board (approval no. 2018LWKYS-06).

\section{Patient consent for publication}

Not applicable. 


\section{Competing interests}

The authors declare that they have no competing interests.

\section{References}

1. Munro MG: Classification and reporting systems for adenomyosis. J Minim Invasive Gynecol 27: 296-308, 2020.

2. Yu O, Schulze-Rath R, Grafton J, Hansen K, Scholes D and Reed SD: Adenomyosis incidence, prevalence and treatment: United States population-based study 2006-2015. Am J Obstet Gynecol: doi:10.1016/j.ajog.2020.01.016.

3. Tomassetti C, Meuleman C, Timmerman D and D'Hooghe T: Adenomyosis and subfertility: Evidence of association and causation. Semin Reprod Med 31: 101-108, 2013.

4. Koike N, Tsunemi T, Uekuri C, Akasaka J, Ito F, Shigemitsu A and Kobayashi $\mathrm{H}$ : Pathogenesis and malignant transformation of adenomyosis (review). Oncol Rep 29: 861-867, 2013. (review).

5. Jichan Nie, Xishi Liu and Guo SW: Promoter hypermethylation of progesterone receptor isoform B (PR-B) in adenomyosis and its rectification by a histone deacetylase inhibitor and a demethylation agent. Reprod Sci 17: 995-1005, 2010.

6. Morelli M, Rocca ML, Venturella R, Mocciaro R and Zullo F: Improvement in chronic pelvic pain after gonadotropin releasing hormone analogue (GnRH-a) administration in premenopausal women suffering from adenomyosis or endometriosis: A retrospective study. Gynecol Endocrinol 29: 305-308, 2013.

7. Bergeron C, Amant F and Ferenczy A: Pathology and physiopathology of adenomyosis. Best Pract Res Clin Obstet Gynaecol 20: 511-521, 2006.

8. Grow DR and Filer RB: Treatment of adenomyosis with long-term GnRH analogues: A case report. Obstet Gynecol 78: 538-539, 1991.

9. Saremi A, Bahrami H, Salehian P, Hakak N and Pooladi A Treatment of adenomyomectomy in women with severe uterine adenomyosis using a novel technique. Reprod Biomed Online 28: 753-760, 2014.

10. Pepas L, Deguara C and Davis C: Update on the surgical management of adenomyosis. Curr Opin Obstet Gynecol 24: 259-264, 2012.

11. Taran FA, StewartEA and Brucker S: Adenomyosis: Epidemiology, risk factors, clinical phenotype and surgical and interventional alternatives to hysterectomy. Geburtshilfe Frauenheilkd 73: 924-931, 2013.

12. Hu SH, Cao BL, Liu X, et al: Drug use law of traditional chinese medicine in treating endometriosis. West J Tradit Chin Med 31: 87-90, 2018 (In Chinese).

13. Wang Y, Wei SB and Wei SB: The second stage therapy of TCM in treating 120 cases of adenomyosis of stagnation pattern of heat-dampness. West J Tradit Chin Med 30: 1-3, 2017 (In Chinese).

14. Liu Z, Li YH, Sun J, et al: Study of usages of Shixiao powder by ancient literature. J Ethnomedicine Ethnopharmacy 27: 7-10, 2018.

15. Zhou J and Qu F: Treating gynaecological disorders with traditional Chinese medicine: A review. Afr J Tradit Complement Altern Med 6: 494-517, 2009.

16. Flower A, Liu JP, Lewith G, Little P and Li Q: Chinese herbal medicine for endometriosis. Cochrane Database Syst Rev 5: CD006568, 2012

17. Cheng C, Gui T, Huang MH, et al. Treatment of adenomyosis patients by bushen huoxue sanyu decoction: A clinical study. CJITWM 34(11): 1302-1305, 2014.

18. Zhang L and Shi YP: Efficacy observation of ultrasonic conductometric acupoint penetration of Wenhua Zhitong recipe for adenomyosis patients. CJITWM 36 (1): 54-58, 2016 (In Chinese)

19. Chen Y, Zhu B, Zhang H, Liu X and Guo SW: Epigallocatechin-3-gallate reduces myometrial infiltration, uterine hyperactivity, and stress levels and alleviates generalized hyperalgesia in mice induced with adenomyosis. Reprod Sci 20 : 1478-1491, 2013

20. Mao X, Wang Y, Carter AV, Zhen X and Guo SW: The retardation of myometrial infiltration, reduction of uterine contractility, and alleviation of generalized hyperalgesia in mice with induced adenomyosis by levo-tetrahydropalmatine (1-THP) and andrographolide. Reprod Sci 18: 1025-1037, 2011.

21. Liu X and Guo SW: Valproic acid alleviates generalized hyperalgesia in mice with induced adenomyosis. J Obstet Gynaecol Res 37: 696-708, 2011.
22. Mehasseb MK, Bell SC and Habiba MA: The effects of tamoxifen and estradiol on myometrial differentiation and organization during early uterine development in the CD1 mouse. Reproduction 138: 341-350, 2009.

23. Zhang X, Yuan H, Deng L, Hu F, Ma J and Lin J: Evaluation of the efficacy of a danazol-loaded intrauterine contraceptive device on adenomyosis in an ICR mouse model. Hum Reprod 23: 2024-2030, 2008.

24. Kida H: Histological analysis of spontaneous adenomyosis-like changes in recombinant inbred mouse uterus (SMXA mouse)--a novel animal model for adenomyosis. Nihon Sanka Fujinka Gakkai Zasshi 46 (4): 323-330, 1994 (In Japanese).

25. YamadaH,ShimadaSandFujimotoS:Endometriosis-adenomyosis mouse model induced by transvaginal pituitary transplantation. Nihon Rinsho 59 (Suppl 1): 221-224, 2001 (In Japanese).

26. Li Y, Zhang SF, Zou SE, Xia X and Bao L: Accumulation of nerve growth factor and its receptors in the uterus and dorsal root ganglia in a mouse model of adenomyosis. Reprod Biol Endocrinol 9: 30, 2011.

27. Li X, Liu X and Guo SW: Clinical profiles of 710 premenopausal women with adenomyosis who underwent hysterectomy. J Obstet Gynaecol Res 40: 485-494, 2014.

28. Cirpan T, Yeniel O, Ulukus M, Ozbal A, Gundem G, Ozsener S, Mete Itil I and Zekioglu O: Clinical symptoms and histopathological findings in subjects with adenomyosis uteri. Clin Exp Obstet Gynecol 35: 48-53, 2008.

29. Shrestha A and Sedai LB: Understanding clinical features of adenomyosis: A case control study. Nepal Med Coll J 14: 176-179, 2012.

30. Nie J, Liu X, Guo SW. Immunoreactivity of oxytocin receptor and transient receptor potential vanilloid type 1 and its correlation with dysmenorrhea in adenomyosis. Am J Obstet Gynecol 202: 346 e1-8, 2010

31. Guo SW, Mao X, Ma Q and Liu X: Dysmenorrhea and its severity are associated with increased uterine contractility and overexpression of oxytocin receptor (OTR) in women with symptomatic adenomyosis. Fertil Steril 99: 231-240, 2013.

32. Taran FA, Weaver AL, Coddington CC and Stewart EA: Understanding adenomyosis: A case control study. Fertil Steril 94: 1223-1228, 2010.

33. Chapman CR, Tuckett RP and Song CW: Pain and stress in a systems perspective: Reciprocal neural, endocrine, and immune interactions. J Pain 9: 122-145, 2008.

34. Li SP, Zhao J and Yang B: Strategies for quality control of Chinese medicines. J Pharm Biomed Anal 55: 802-809, 2011

35. Reagan-Shaw S, Nihal M and Ahmad N: Dose translation from animal to human studies revisited. FASEB J 22: 659-661, 2008.

36. Ascher SM, Imaoka I and Lage JM: Tamoxifen-induced uterine abnormalities: The role of imaging. Radiology 214: 29-38, 2000.

37. Mori T, Yamasaki S, Masui F, Matsuda M, Sasabe H and Zhou YF: Suppression of the development of experimentally induced uterine adenomyosis by a novel matrix metalloproteinase inhibitor, ONO-4817, in mice. Exp Biol Med (Maywood) 226: 429-433, 2001

38. Gui T, Chen C, Zhang Z, Tang W, Qian R, Ma X, Cao P and Wan G: The disturbance of TH17-Treg cell balance in adenomyosis. Fertil Steril 101: 506-514, 2014.

39. Yan Li, Shaofen Zhang, Xian Xia and Enshi Zhou: Establishment of an ICR mouse model of adenomyosis by oral administration of Tamoxifen. Acta laboratorium animalis scientia sinica 17 (5): 345-350, 2009 (In Chinese).

40. Bird CC, McElin TW and Manalo-Estrella P: The elusive adenomyosis of the uterus--revisited. Am J Obstet Gynecol 112: 583-593, 1972

41. Emge LA: The elusive adenomyosis of the uterus. Its historical past and its present state of recognition. Am J Obstet Gynecol 83: 1541-1563, 1962.

42. Mechsner S, Bartley J, Loddenkemper C, Salomon DS, Starzinski-Powitz A and Ebert AD: Oxytocin receptor expression in smooth muscle cells of peritoneal endometriotic lesions and ovarian endometriotic cysts. Fertil Steril 83 (Suppl 1): 1220-1231, 2005.

43. Szallasi A: Vanilloid (capsaicin) receptors in health and disease. Am J Clin Pathol 118: 110-121, 2002.

44. Lu AP, Jia HW, Xiao C and Lu QP: Theory of traditional Chinese medicine and therapeutic method of diseases. World J Gastroenterol 10: 1854-1856, 2004.

45. Garcia L and Isaacson K: Adenomyosis: Review of the literature. J Minim Invasive Gynecol 18: 428-437, 2011. 
46. Taran FA, Weaver AL, Coddington CC and Stewart EA: Characteristics indicating adenomyosis coexisting with leiomyomas: A case-control study. Hum Reprod 25: 1177-1182, 2010

47. Levgur M, Abadi MA and Tucker A: Adenomyosis: Symptoms, histology, and pregnancy terminations. Obstet Gynecol 95: 688-691,2000.

48. Greaves P and White IN: Experimental adenomyosis. Best Pract Res Clin Obstet Gynaecol 20: 503-510, 2006.

49. Zhu B, Chen Y, Zhang H, Liu X and Guo SW: Resveratrol reduces myometrial infiltration, uterine hyperactivity, and stress levels and alleviates generalized hyperalgesia in mice with induced adenomyosis. Reprod Sci 22: 1336-1349, 2015.

50. Moriyama T, Higashi T, Togashi K, Iida T, Segi E, Sugimoto Y, Tominaga T, Narumiya S and Tominaga M: Sensitization of TRPV1 by EP1 and IP reveals peripheral nociceptive mechanism of prostaglandins. Mol Pain 17: 1:3, 2005.

51. Nie J and Liu X: Quercetin alleviates generalized hyperalgesia in mice with induced adenomyosis. Mol Med Rep 16: 5370-5376, 2017.
52. Kurtais Y, Tur BS, Elhan AH, Erdogan MF and Yalçin P: Hypothalamic-pituitary-adrenal hormonal responses to exercise stress test in patients with rheumatoid arthritis compared to healthy controls. J Rheumatol 33 (8): 1530-1537, 2006.

53. Otake T, Ashihara M, Nishino J, Kato K, Fukaya S and Yoshida S: Stressors and rheumatoid arthritis: Changes in stressors with advances in therapeutic agents. Rheumatol Int 33: 887-891, 2013.

54. Cuevas M, Flores I, Thompson KJ, Ramos-Ortolaza DL, Torres-Reveron A and Appleyard CB: Stress exacerbates endometriosis manifestations and inflammatory parameters in an animal model. Reprod Sci 19: 851-862, 2012.

55. Ji GQ, Xu M, Yin H, et al: Study on Shixiaosan Granule on Toxicity in Rats. Acta Chinese Medicine 6: 985-988, 2017.

56. Li Q, Xie P, Bai CX, Cui JY, Jiang C and Wu YS: Mechanisms of Fufang Shixiao formula for experimental primary dysmenorrhea. Zhongguo Zhong Xi Yi Jie He Za Zhi 36: 1087-1090, 2016. 